\title{
Análisis del proceso constructivo y variabilidad de parámetros geotécnicos en la capacidad de carga de micropilotes usando elementos finitos aleatorios
}

\section{Analysis of the construction process and geotechnical parameters variability on the loading capacity of micropiles using random finite elements}

Fecha de entrega: 13 de marzo 2012

Fecha de aceptación: 11 de mayo 2012

\author{
Christian Mendoza ${ }^{1}$, Alfonso M. Ramos ${ }^{2}$, Renato Cunha ${ }^{3}$, Arcesio \\ Lizcano ${ }^{4}$ \\ ${ }^{1}$ Departamento de Ingeniería Civil y Ambiental, Universidad de Brasilia, CEP 70910-900 - Prédio SG-12, Brazil, \\ ccmb80@aluno.unb.br \\ ${ }^{2}$ Instituto Geofísico, Facultad de Ingeniería, Pontificia Universidad Javeriana, Cra 7 No 40-62, Bogotá, Colombia, \\ a-ramos@javeriana.edu.co \\ ${ }^{3}$ Departamento de Ingeniería Civil y Ambiental, Universidad de Brasilia, CEP 70910-900 - Prédio SG-12, Brazil, rpcunha@unb.br \\ ${ }^{4}$ SRK Consulting, Vancouver, British Columbia, Canadá, (anteriormente en Estructuras y Geotecnia, Universidad de los Andes, \\ Bogotá, Colombia) alizcano@srk.com
}

En la práctica normal de ingeniería de cimentaciones se calcula la capacidad de carga de un micropilote sin tener en cuenta que el proceso constructivo puede afectar la geometría del problema. En este trabajo se cuantifica la influencia tanto del proceso constructivo como de la variabilidad inherente de las propiedades del suelo en la capacidad de carga de un micropilote autoperforante e inyectado, a través de simulaciones de la excavación con elementos finitos aleatorios. Se obtiene que al no tener en cuenta el método constructivo del micropilote y la variabilidad de las propiedades del suelo, se sobreestima en un factor entre dos y tres la capacidad de carga por fuste. Por lo tanto, se presenta una explicación racional al factor de seguridad que se debe aplicar para obtener la capacidad de carga de diseño. Finalmente, se presenta un procedimiento para generar cartas de diseño de micropilotes autoperforantes materializado en uno que resalta la importancia de considerar la variabilidad inherente de las propiedades del suelo y el método constructivo.

Palabras claves: método de elementos finitos, simulaciones Montecarlo, elastoplasticidad, micropilotes
In the normal practice of foundation engineering the bearing capacity of micropiles is calculated without taking into account that the construction processes may affect the problem geometry. This work quantifies the influence of both, construction processes and the inherent variability of soil properties in the bearing capacity of a selfbored and gravitationally grouted micropile by means of simulations with random finite element method. When the construction process and the inherent variability are not taken into account, the shaft bearing capacity is overestimated in a factor between two and three. Therefore, a rational explanation of the safety factor is presented in order to calculate the design capacity load. Lastly, a procedure to generating design charts for selfbored and grouted micropiles is presented and its application shows the importance of considerate the inherent variability of the soil properties and the construction process.

Keywords: finite element method, Montecarlo simulations, elastoplasticity, micropiles

que el tubo de perforación sirve como refuerzo interno del micropilote, además de su alto rendimiento en construcción (alrededor de 10 minutos). Por otro lado, una desventaja
Anker, está siendo muy usado en la ciudad de Bras (Mendoza et al., 2011). Dentro de sus ventajas se tiene 
que tienen estos micropilotes autoperforantes es que en la etapa de perforación las paredes de la excavación tienden a cerrarse, lo que disminuye el diámetro del micropilote, la superficie de contacto entre el micropilote-suelo y consecuentemente la capacidad de carga.

La formulación convencional para calcular la capacidad portante está basada en los teoremas de límite superior o inferior si se usa un modelo constitutivo elastoplástico perfecto (FHWA 2000, Delgado, 2001). Dado lo anterior, el problema de esfuerzo deformación del suelo está desacoplado. Es decir, no es posible obtener estimativos de desplazamientos en la masa del suelo de cimentación debido a los incrementos de carga. Los análisis de elementos finitos aplicados a geotecnia son una potente herramienta que permite asegurar equilibrio de esfuerzos y compatibilidad de deformaciones a través de un modelo constitutivo. Sin embargo, en la práctica convencional de consultoría no se acostumbra a utilizar análisis de elementos finitos debido, entre otros, a los siguientes aspectos. 1) las inversiones en exploración del subsuelo y principalmente en el desarrollo de ensayos de laboratorio son importantes dado que es necesario estimar los valores de los parámetros de los modelos constitutivos usados en los elementos finitos. 2) se podría argumentar que los factores de seguridad que se aplican en el cálculo de la capacidad de carga convencional tienen incluidos indirectamente una cuantificación de la incertidumbre (en el proceso constructivo y en los parámetros geotécnicos del suelo), por lo que el sobresfuerzo en recursos que conllevan los análisis de elementos finitos no son necesarios.

Este trabajo presenta el uso del Método de Elementos Finitos MEF como herramienta para solucionar el problema acoplado de esfuerzo deformación en la etapa de construcción de micropilotes. Los desplazamientos de la pared de la excavación obtenidos del análisis de elementos finitos servirán como insumo para afectar la geometría de la formulación convencional de capacidad portante (ecuación 2). De esta manera se integrará el proceso constructivo a la formulación convencional de la capacidad de carga. Se usará el modelo constitutivo elastoplástico de Mohr Coulomb en el MEF debido a que el número de parámetros es bajo y además es el de más amplio uso en la comunidad geotécnica. En este trabajo se estudia la influencia en la capacidad de carga por fuste al ser el parámetro de diseño imperante en este tipo de cimentación. Adicionalmente, se tendrá en cuenta la influencia de la variabilidad inherente de los parámetros geomecánicos a través de la incorporación del carácter estocástico en el MEF, similar a lo que han realizado Papaioannou y Straub (2012), Fellin et al., (2010), Baroth y Malecot (2010), Kamruzzaman y Lye (2008).

En el artículo se muestra las características y método constructivo del micropilote llamado Alluvial Anker. Luego se presenta el modelo constitutivo elastoplástico de Mohr Coulomb que será usado en los análisis de MEF y la geometría del modelo del MEF y su parametrización. A continuación se presentan los parámetros del modelo constitutivo que serán adoptados como variables aleatorias en la modelación con el MEF. Se presenta el tipo de las funciones de distribución de probabilidad junto con los parámetros que las caracterizan y la manera como se integró el carácter estocástico de las variables aleatorias al modelo del MEF, a través de simulaciones de Montecarlo. Luego se presenta un breve resumen de la teoría de capacidad portante convencional ya que ésta es la formulación que se usará para incluir los resultados de los análisis del MEF estocásticos en la capacidad de carga de pilotes. Finalmente se presentan los resultados con su respectivo análisis e implicaciones prácticas y se resaltan los aspectos más importantes del análisis de resultados.

\section{Micropilote Alluvial Anker}

El micropilote 1lamado Alluvial Anker puede alcanzar diámetros de aproximadamente $13 \mathrm{~cm}$, es autoperforado por rotación con una broca en forma de flecha la cual va unida a la punta de un tubo de acero de $62.5 \mathrm{~mm}$ de diámetro conectado a una máquina perforadora (Figuras 1 y 2). Por medio de un mandril que inyecta constantemente líquido refrigerante a la punta cortante. Este líquido puede ser lechada de cemento o agua, dependiendo del tipo de suelo. Después de terminado el proceso de perforación con el líquido refrigerante a la profundidad deseada, se deja el tubo como refuerzo del pilote y se procede a la inyección por gravedad de lechada rica en cemento (si el líquido refrigerante fue agua). La Figura 2 ilustra la secuencia constructiva del micropilote donde al contrario de lo usual en micropilotes, no se inyecta a altas presiones. 


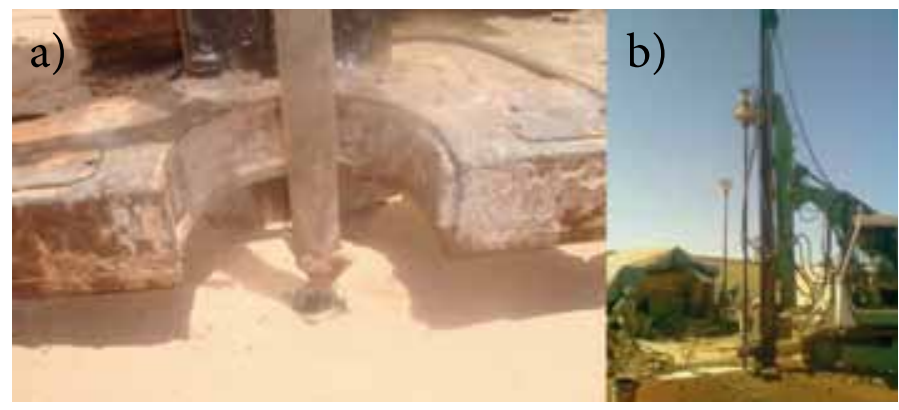

Figura 1: a) Punta y tubo de refuerzo autoperforante para micropilote Anker y b) máquina de perforación
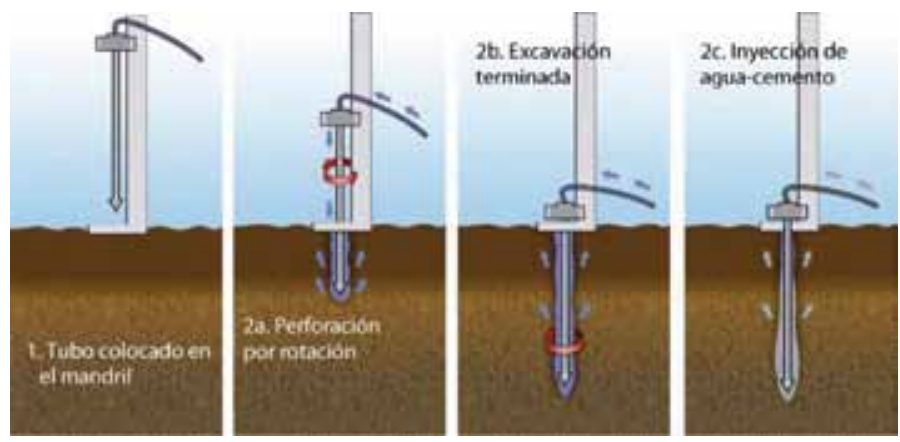

Figura 2: Secuencia constructiva del micropilote Alluvial Anker (Mendoza et al., 2011)

\section{Modelo constitutivo}

Los análisis se realizaron para un suelo residual con muy baja cohesión $(c<10 \mathrm{kPa})$ por lo que se despreció el aporte de la cohesión efectiva considerándose sólo la fricción del suelo en la resistencia al corte. El suelo es poroso tropical, muy blando y tiene un índice de vacíos $e=2$, un coeficiente de permiabilidad $K$ entre $10^{-3}$ y $10^{-4} \mathrm{~m} / \mathrm{s}$ y un número de golpes/pies igual a 2 en el ensayo SPT. El modelo constitutivo seleccionado para la simulación con elementos finitos del comportamiento esfuerzo deformación del suelo es del tipo elastoplástico con criterio de falla de Mohr Coulomb. Este modelo es ampliamente usado en la geotecnia práctica al poseer sólo 4 parámetros que tienen su correspondiente explicación física. El uso de modelos constitutivos más complejos que incorporen por ejemplo el cambio de rigidez del suelo con la profundidad requieren de más de 4 parámetros, aumentando la incertidumbre aleatoria. Esto significa que las probabilidades de falla serán mayores. En el rango elástico la relación entre el tensor de esfuerzos y deformaciones es de tipo lineal, siendo los parámetros elásticos el módulo de Young y la relación de Poisson. Dicho comportamiento es válido hasta que la trayectoria de esfuerzos alcanza la envolvente de fluencia, momento en el que empiezan a generarse deformaciones plásticas (Figura 3). La envolvente de fluencia es del tipo Mohr Coulomb, por lo que está asociada a los otros dos parámetros del suelo, el ángulo de fricción del suelo $\phi$ y la cohesión del suelo $c$. Dichos parámetros se pueden obtener de ensayos de laboratorio convencionales como corte directo o triaxiales.
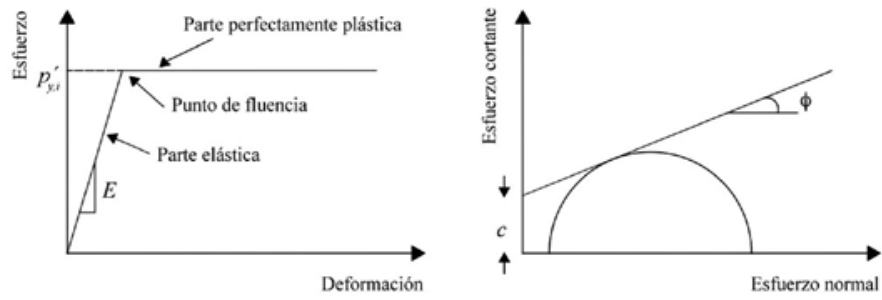

Figura 3: a) Relación esfuerzo deformación en el modelo constitutivo elastoplástico y b) criterio de falla de Mohr Coulomb usado en las simulaciones de elementos finitos

\section{Modelo de elementos finitos}

Se realizaron 4 modelos diferentes de elementos finitos variando la profundidad de la excavación usando el programa ABAQUS, bajo la suposición de un horizonte homogéneo de suelo (Tandjiria et al., 2000; Cherubini, 2000). Se utiliza el MEF para simular el proceso de excavación previo a la inyección de lechada. La parametrización de la geometría del modelo se realizó en función de la longitud y diámetro del pilote. Se dispuso una longitud adicional de la mitad de la profundidad para micropilotes de largo $\mathrm{L}=12 \mathrm{~m}, 20 \mathrm{~m}, 40 \mathrm{~m}, 60 \mathrm{~m}$, con un ancho del modelo de 30 veces el diámetro teórico del pilote $\mathrm{D}=13 \mathrm{~cm}$ (Helwany, 2007). Los valores de $\mathrm{L}=40 \mathrm{y}$ $60 \mathrm{~m}$ se usaron con el fin de completar el análisis numérico sabiendo que tal vez no tendrán aplicación en la práctica. El análisis se realizó en condiciones axial simétricas de carga (Figura 4). Los elementos seleccionados para el mallado son del tipo CAX8P (Continuo, Axisimétrico, 8 nodos, con presión de poros). En la Figura 4 se presenta la distribución de los elementos.

Una vez creada la geometría del modelo de excavación se aplicaron dos pasos de análisis. El paso de carga geostático induce los esfuerzos a los que está sometido el suelo, generando una distribución lineal con la profundidad, como se ilustra en la Figura 5. Posteriormente se crea el paso de excavación, que simula simplificadamente 
el proceso de perforación por medio del apagado de los elementos delimitados. Debido a que la perforación se realiza rápidamente entre 2 y 4 minutos, pero en un suelo permeable, se justifica el uso de un modelo constitutivo que no considera el efecto del tiempo. El esquema de pasos es ilustrado a la derecha de la Figura 5.
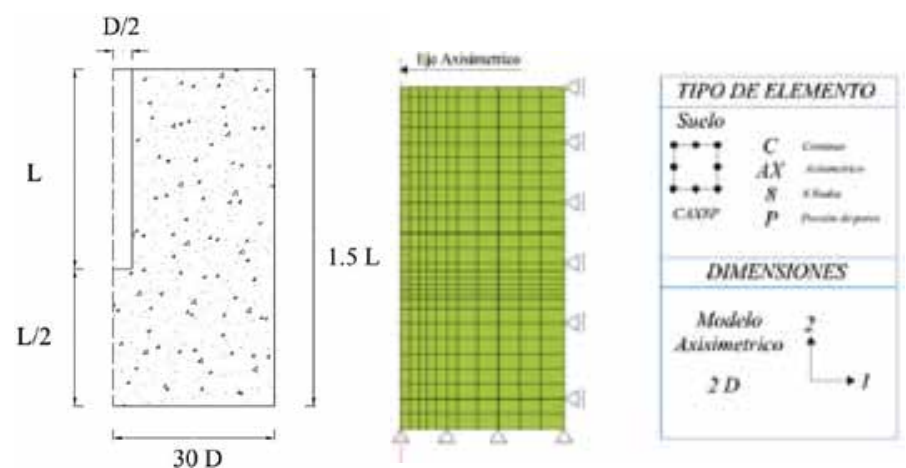

Figura 4: Parametrización del modelo, condiciones de borde y mallado del modelo
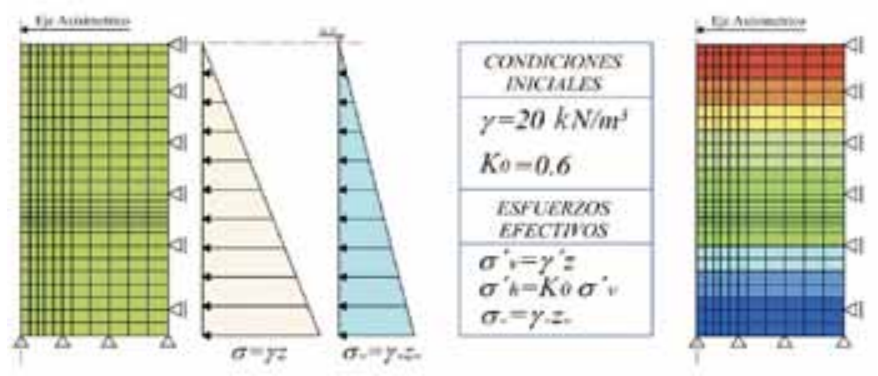

a)
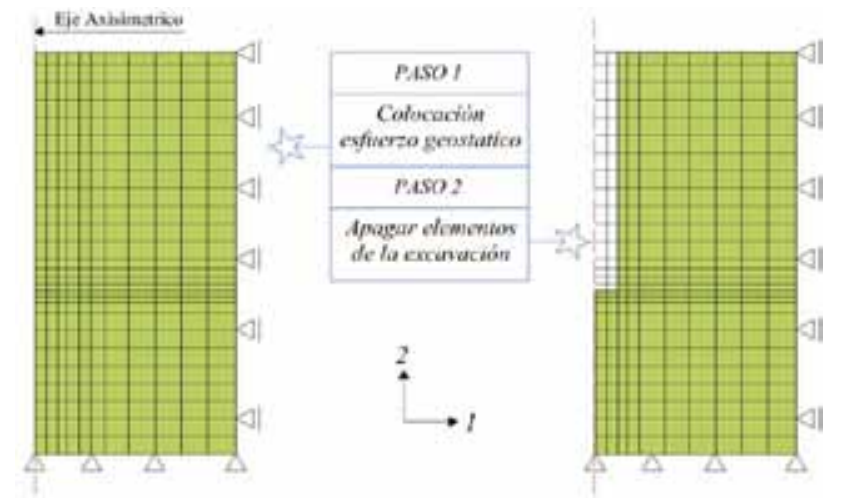

b)

Figura 5: a) Esfuerzos iniciales en el modelo de elementos finitos y b) excavación del micropilote en el modelo

\section{MEF con parámetros estocásticos}

Para capturar la incertidumbre inherente de las variables del modelo constitutivo en el análisis geomecánico por medio del MEF, se usó el método de simulación numérica denominado Montecarlo. En este caso, las soluciones de la función de distribución de probabilidad de tipo analítico no están disponibles dado que el proceso es altamente no lineal (modelo constitutivo y geometría). Las simulaciones de Montecarlo es un proceso numérico de cálculo repetido de un operador matemático en el que variables dentro del operador son aleatorias o contienen incertidumbre con funciones de distribución preescritas. Los resultados numéricos de cada repetición del proceso numérico se pueden considerar como una muestra de la solución verdadera, de forma análoga a una muestra observada o a un experimento físico (Ang y Tang, 2007). En este caso, el operador es el MEF por lo que de esta manera se incluye la incertidumbre inherente o aleatoria de los parámetros geomecánicos (Sentz y Ferson, 2011). Para cada modelo de elementos finitos se realizaron 10.000 ejecuciones, variando los parámetros del modelo constitutivo en cada punto de Gauss. Para realizar tal acción, fue necesario escribir una rutina en lenguaje phyton que permitiera automatizar la escritura del archivo de entrada, la ejecución de cada modelo y la adquisición del archivo de respuesta en el programa ABAQUS. Las funciones de distribución de probabilidad de los parámetros geomecánicos se incluyen en la siguiente sección. Las simulaciones de Montecarlo generan una serie que puede ser usada para estimar la distribución de probabilidades de las variables de respuestas (Zevgolis y Bourdeau, 2010; Harahap y Wong, 2008; Prada et al., 2011).

\section{Incertidumbre aleatoria de los parámetros del modelo constitutivo}

Dado que se reconoce el hecho que las propiedades geomecánicas del terreno tienen una variabilidad inherente, y esa variabilidad afecta los resultados del proceso constructivo de micropilotes autoperforantes e inyectados, se caracterizó cada parámetro del modelo constitutivo elastoplástico con el criterio de falla de Mohr Coulomb siguiendo las consideraciones mostradas en la Tabla 1.

Dado que las variables seleccionadas como aleatorias en la Tabla 1 no pueden tomar valores negativos, una alternativa frecuente es usar la distribución tipo log-normal para mapear variables del tipo geomecánico (Griffiths et al., 2005; Papaioannou y Straub, 2012; Huang et al., 2010). 
Los valores medios y del coeficiente de variación COV de las variables de la Tabla 1 son típicos del suelo residual de Brasilia. Papaioannou y Straub (2012) usan un COV igual a 0.25 para el módulo de Young, en tanto que Kuo et al., (2004) usan 0.2. Baecher y Christian (2003) reportan COV que varían entre 0.02 y 0.4 para ángulos de fricción.

Tabla 1: Valores de las variables aleatorias usadas en el modelo constitutivo elastoplástico

\begin{tabular}{|l|c|c|c|}
\hline Parámetro & media & $\mathrm{COV}$ & $\begin{array}{c}\text { Función de } \\
\text { distribución de } \\
\text { probabilidad }\end{array}$ \\
\hline Ángulo de fricción $\phi,\left[^{\circ}\right]$ & 20 & 0.30 & lognormal \\
\hline Módulo de Young E, $\mathrm{kPa}$ & 1500 & 0.40 & lognormal \\
\hline Relación de Poisson $v$ & 0.3 & 0.40 & lognormal \\
\hline
\end{tabular}

COV: coeficiente de variación

Con base en las funciones de distribución teóricas seleccionadas, se procedió a la generación de 10000 números aleatorios para cada parámetro del modelo constitutivo (Tabla 1). Los diagramas de frecuencia de cada parámetro se presentan en la Figura 6.
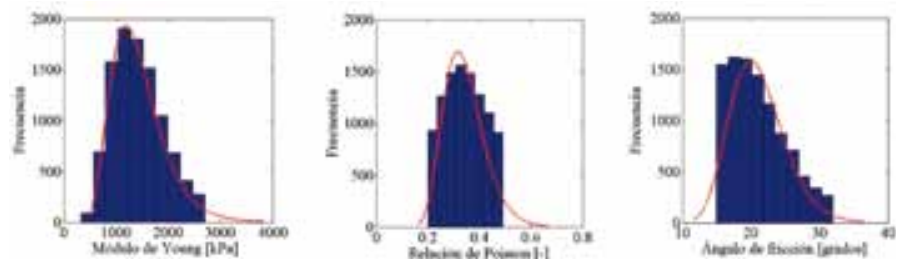

Figura 6: Diagramas de frecuencia y función de distribución de probabilidad de los parámetros del modelo constitutivo elastoplástico de Mohr Coulomb.

La generación de valores aleatorios para el ángulo de fricción y el módulo de elasticidad se limitan a valores de más o menos una desviación estándar y la relación de Poisson se truncó para que el máximo valor fuera de 0.49 y el menor valor equivale a dos desviaciones estándar. Dichas consideraciones se basan en que los parámetros del modelo constitutivo estuvieran en un rango tal que siempre presenten sentido físico. La incertidumbre del peso unitario del suelo no se considera debido al hecho que su rango de variabilidad es pequeño en comparación al del módulo de
Young y el ángulo de fricción (Phoon y Kulhawy, 1999; Luo et al., 2012; Zevgolis y Bourdeau, 2010).

\section{Capacidad portante para cimentaciones profundas}

Los resultados de los análisis de elementos finitos entregan los desplazamientos de las paredes de la excavación, que a su vez afectan el componente geométrico de la formulación clásica de la capacidad de carga para un pilote. La capacidad de carga de un pilote está dada por la suma de la resistencia por punta del pilote y la fricción entre el suelo y el pilote (resistencia por fuste). En micropilotes y en suelos granulares la componente de capacidad de carga más importante es la que se desarrolla por fricción en el fuste (Poulos y Davis, 1980). La resistencia por fricción se obtiene por medio del criterio de falla de Mohr-Coulomb,

$$
\tau_{\mathrm{a}}=c_{a}+\sigma_{\mathrm{n}}^{\prime} \tan \delta
$$

donde $c_{a}$ es la adherencia ente el pilote y el suelo, $\sigma_{\mathrm{n}}^{\prime}=k_{s} \sigma_{\mathrm{v}}$, es el esfuerzo efectivo normal a la superficie del pilote, $k_{s}$

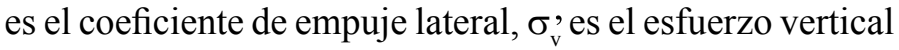
efectivo y $\delta$ ' es el ángulo de fricción efectivo entre el suelo y el pilote. Por medio de la integración de la resistencia al corte $\tau_{\mathrm{a}}$ en el área expuesta a la fricción se obtiene la resistencia de fuste.

$$
f_{s u}=\int_{0}^{\mathrm{L}} \mathrm{C}\left(c_{a}+k_{s} \sigma_{\mathrm{v}}^{\prime} \tan \delta^{\prime}\right) d z
$$

donde $C$ es el perímetro del pilote y $\mathrm{L}$ es la longitud del pilote. Se ha reportado en la literatura valores de $k_{s}$ que varían de 0.7 a 3.0 (Delgado, 2001). Burland (1973) propone una expresión para la resistencia por fricción en arcillas remoldeadas en condición drenada,

$$
f_{s}=\beta \sigma_{\mathrm{v}}
$$

donde el factor $\beta$ está relacionado con el ángulo de fricción drenado entre el suelo y el pilote.

$$
\beta=k_{s} \tan \delta
$$

Se ha observado en pruebas de carga en arcillas normalmente consolidadas, que el factor $\beta$ puede obtener un valor máximo de 0.4 (Delgado, 2001).

En el presente trabajo se adopta el método $\beta$ debido a que se asume que los micropilotes desarrollan una resistencia de fuste drenada debido principalmente a los altos valores 
del coeficiente de permeabilidad $k$ del suelo residual poroso (porosidad $n \approx 65 \%$ ) de la ciudad de Brasilia, con valores de $k$ entre $10^{-3}$ y $10^{-4} \mathrm{~m} / \mathrm{s}$.

\section{Resultados}

Los resultados de los análisis se presentan en dos partes. La primera es el análisis de la variabilidad resultante de los desplazamientos horizontales de la excavación para las 4 profundidades de micropilotes y la segunda presenta la influencia del proceso constructivo en la capacidad de carga del micropilote.

\section{Perfiles de desplazamiento}

La Figura 7a presenta el valor medio del perfil de desplazamiento horizontal de la pared de la excavación para las cuatro diferentes profundidades. El perfil promedio se calculó haciendo el promedio aritmético de las 10.000 ejecuciones de Montecarlo. A mayor profundidad, aumenta el desplazamiento de la pared en una forma que es claramente no lineal. El desplazamiento promedio máximo para cada profundidad se presenta en el fondo de la excavación, con valores que varían de un poco más de 1 $\mathrm{cm}$, hasta casi $2.3 \mathrm{~cm}$ para longitudes de excavación de 12 $\mathrm{m}$ a $60 \mathrm{~m}$, respectivamente.

La Figura $7 \mathrm{~b}$ presenta el coeficiente de variación del desplazamiento para cada profundidad de excavación. El comportamiento en general es similar para todas las profundidades. A pesar que la incertidumbre de los parámetros geomecánicos se mantiene constante con la profundidad en todos los casos (Tabla 1), el coeficiente de variación del perfil de desplazamiento disminuye con la profundidad. El gradiente del coeficiente de variación es mayor en la superficie. Dentro del primer metro de profundidad, el coeficiente de variación disminuye abruptamente y la tendencia es la estabilización a grandes profundidades. Las implicaciones prácticas del análisis anterior es que la variabilidad inherente de las propiedades geomecánicas tiene un mayor impacto en la incertidumbre de los desplazamientos calculados próximos a la superficie. Dado lo anterior, a pesar que los valores calculados del desplazamiento son más pequeños en superficie, a su vez son más difíciles de predecir dada su variabilidad. Dicha situación se presenta debido a la falta de restricción de movimiento de los nodos superficiales de los elementos finitos, lo que permite mayor variedad de desplazamientos. Esta situación es concordante con las observaciones de campo. A pesar que se impone que la variabilidad para toda la profundidad sea constante, los resultados indican mayor homogeneidad de la variable de salida en profundidad. Los esfuerzos efectivos en profundidad confinan el suelo de forma tal que limitan los desplazamientos, en tanto que en superficie los esfuerzos son bajos y el suelo tiene mayor libertad de movimiento.

Otro aspecto interesante en la Figura $7 \mathrm{~b}$ es la tendencia a estabilización del coeficiente de variación del desplazamiento en valores que son cercanos a 0.2 para grandes profundidades. El valor de estabilización del COV es menor que cualquiera de las variabilidades impuestas (Tabla 1).

Las Figuras 7a y $7 \mathrm{~b}$ entregan los parámetros de medida central y de dispersión de una función de densidad de probabilidad única de desplazamientos de la excavación condicionados a la profundidad $z, p(d / z)$. Si se aplica el teorema de probabilidad total (Ang y Tang, 2007) a los factores que afectan los desplazamientos $d$ de la pared (parámetros geomecánicos del suelo $\phi ', E, v$ ), se podría estimar la probabilidad del desplazamiento. Siendo estos, insumos para el diseño basado en confiabilidad de excavaciones para cimentaciones profundas. Los subíndices en (5) representan una realización específica de cada variable aleatoria.

$$
\begin{aligned}
& \mathrm{p}\left(\mathrm{d}_{\mathrm{i}, \mathrm{j}, \mathrm{k}, \mathrm{l,m}}\right)=\underbrace{\mathrm{p}\left(\mathrm{d}_{\mathrm{i}} / \mathrm{z}_{\mathrm{j}}\right)}_{\text {Figura } 7} \mathrm{p}\left(\mathrm{z}_{\mathrm{j}}\right)+\mathrm{p}\left(\mathrm{d}_{\mathrm{i}} / \phi_{\mathrm{k}}\right) \underbrace{\mathrm{p}\left(\phi_{\mathrm{k}}\right)}_{\text {Figura } 6}+\mathrm{p}\left(\mathrm{d}_{\mathrm{i}} / \mathrm{E}_{1}\right) \underbrace{\mathrm{p}\left(\mathrm{E}_{1}\right)}_{\text {Figura } 6} \\
& +\mathrm{p}\left(\mathrm{d}_{\mathrm{i}} / \mathrm{v}_{\mathrm{m}}\right) \underbrace{\mathrm{p}\left(v_{\mathrm{m}}\right)}_{\text {Figura } 6}
\end{aligned}
$$

En la Figura 7c se presenta la profundidad normalizada versus el porcentaje de disminución del radio debido al desplazamiento de la pared. La profundidad normalizada se calcula como la profundidad dividida por la profundidad de excavación máxima $(12 \mathrm{~m}, 20 \mathrm{~m}, 40 \mathrm{~m}$ y $60 \mathrm{~m})$. Las abscisas de la Figura 7c se obtienen al dividir la diferencia entre el radio original $(6.5 \mathrm{~cm})$ y el desplazamiento de la pared entre el radio original de la excavación. Se observa que a mayor profundidad de excavación máxima, la 
disminución del radio es mucho mayor. El porcentaje de disminución del radio varía desde un $20 \%$ para la excavación de $12 \mathrm{~m}$, hasta un $37 \%$ para excavaciones de $60 \mathrm{~m}$. Esta disminución del radio de la excavación para micropilotes se verá reflejada en una disminución de la capacidad de carga del micropilote.
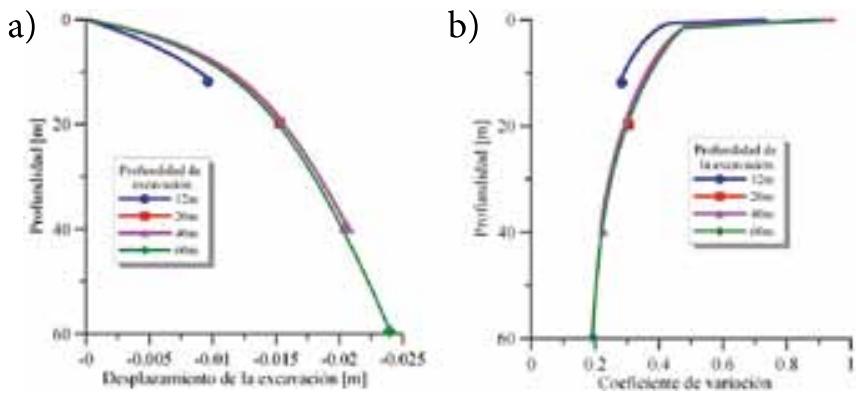

c)

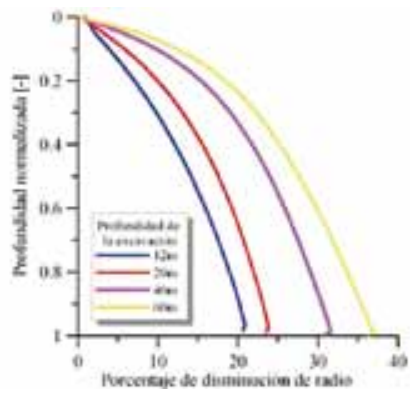

Figura 7: a) Perfil promedio de desplazamiento horizontal de las paredes de la excavación, b) coeficiente de variación del desplazamiento horizontal en función de la profundidad y c) porcentaje de disminución del radio de la excavación por el desplazamiento de la pared en función de la profundidad

En el cálculo convencional de capacidad de carga, no es posibletenerencuentalainfluencia del proceso constructivo. En este caso, la relación entre esfuerzo y deformación está desacoplada. Una alternativa a la dificultad previamente mencionada es afectar el componente geométrico de la formulación convencional dada en la ecuación (1) con los resultados de desplazamiento de las paredes obtenidos del análisis de elementos finitos de la excavación.

\section{Capacidad de carga}

Como se observó en la sección anterior, la variabilidad inherente de las propiedades geomecánicas del terreno influye en los desplazamientos de la pared debido a la perforación. A su vez, dichos desplazamientos generan disminución de la capacidad de carga. El análisis de elementos finitos aleatorio se integró con la formulación convencional de capacidad portante, usando como puente dos aspectos. Primero, los desplazamientos de la pared de la excavación afectan la geometría de la formulación de la capacidad portante (diámetro del micropilote). Segundo, se incluye el ángulo de fricción como una variable aleatoria en el modelo constitutivo (Figura 6) que en el análisis de elementos finitos se usó como insumo para el cálculo de la capacidad portante.

La Figura 8 presenta los histogramas de frecuencia de la capacidad de carga por fuste para las diferentes longitudes de micropilote. La capacidad de carga por fuste se calculó para cada simulación de Montecarlo.
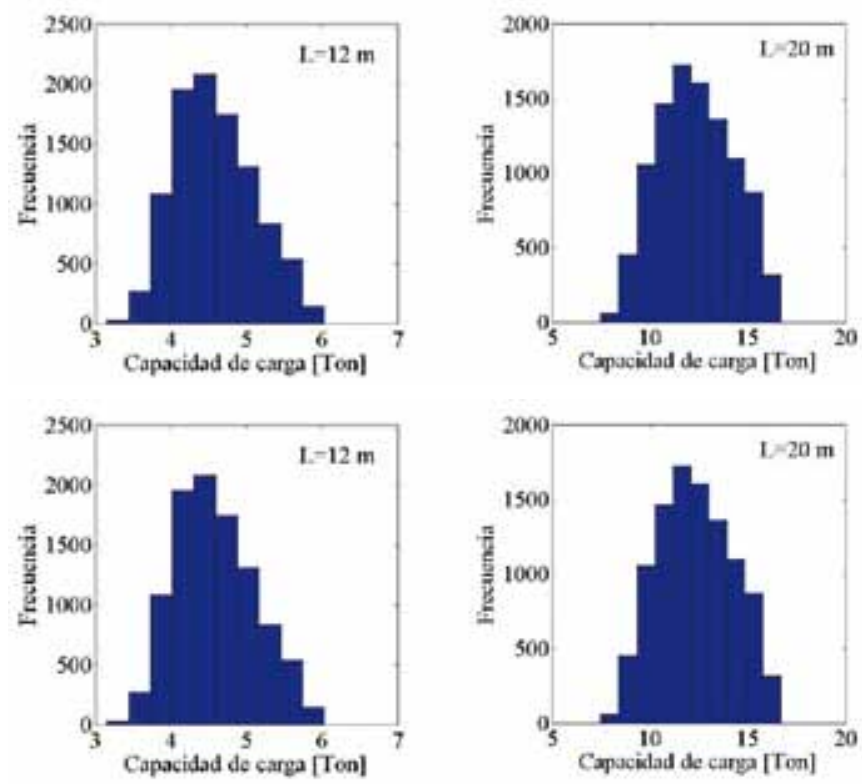

Figura 8: Histogramas de frecuencia de la capacidad de carga para micropilotes de diferentes longitudes L

La Figura 9 presenta las funciones de distribución empírica acumulada de la capacidad de carga por fuste calculadas con los histogramas de frecuencia de la Figura 8. Se observa que la mediana de la capacidad de carga aumenta con la profundidad del micropilote.

La Tabla 2 muestra la capacidad de carga por fuste promedio y la desviación estándar obtenida de las funciones de distribución empírica (Figura 9). Se calcula la capacidad de carga determinística tomando los valores promedios del horizonte mostrado en la Tabla 1. Esta última es la manera convencional de cálculo, donde no se tiene en cuenta la influencia del proceso constructivo (diámetro del pilote constante $d=13 \mathrm{~cm}$ ). Adicionalmente se determina 
el porcentaje de pérdida de capacidad de carga por fuste si se tiene en cuenta el efecto del menor diámetro del micropilote debido al proceso constructivo. En la Figura 7c se observó que el porcentaje de disminución promedio del radio del pilote debido a los desplazamientos generados por el proceso constructivo (excavación) presenta valores máximos de $20 \%$ y $37 \%$ para las profundidades de $20 \mathrm{~m}$ y $60 \mathrm{~m}$, respectivamente. Estos porcentajes de disminución no se reflejan directamente en la pérdida de capacidad de carga por fuste, que están entre 53 y $62 \%$ de la capacidad de carga sin tener en cuenta el proceso constructivo (Tabla 2). Porcentualmente, la disminución de capacidad de carga es mayor que la disminución del radio de micropilote. Wang y Sitar (2011) reportan una capacidad de carga de 25 toneladas para un pilote de $6 \mathrm{~m}$ de longitud y $0.7 \mathrm{~m}$ de diámetro en un suelo arcillo-arenoso muy rígido.
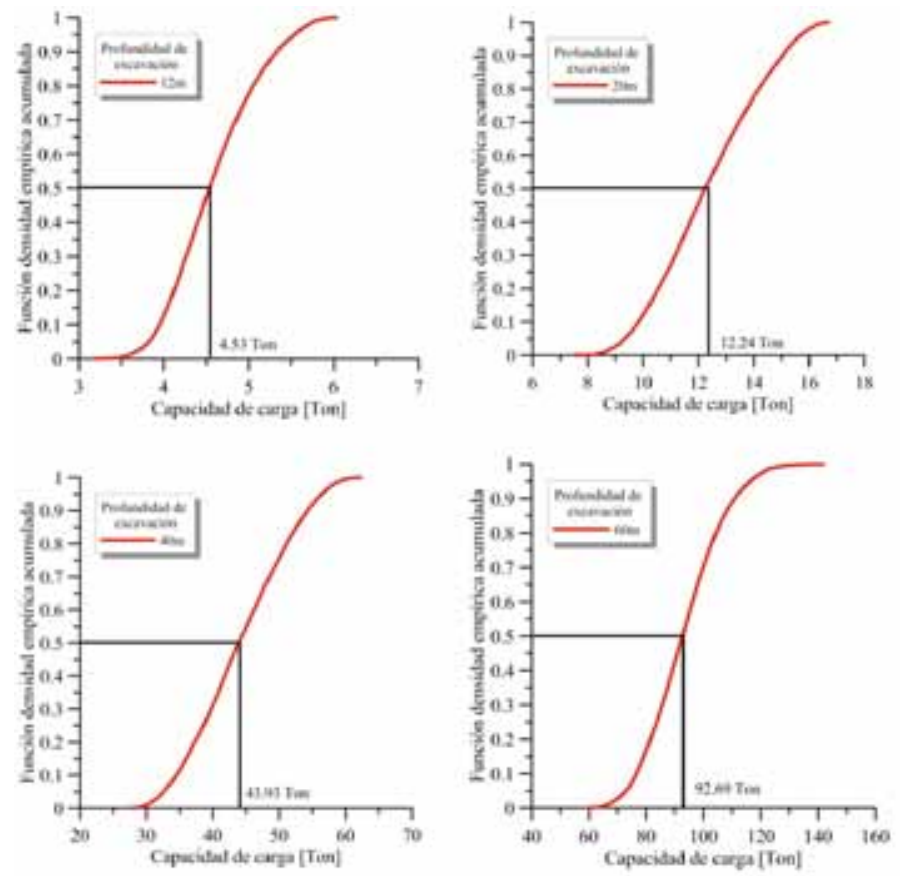

Figura 9: Función de distribución empírica acumulada de la capacidad de carga por fuste para diferentes profundidades. Mediana de la capacidad de carga.

En la Tabla 2 se incluye la capacidad de carga en la base determinista (calculada con los valores medios de la Tabla 1). Al comparar los resultados determinísticos de capacidad de carga por fuste y por punta, se ratifica el hecho que el aporte principal a la resistencia del micropilote se debe a la fricción por fuste del micropilote.
Tabla 2: Capacidad de carga media y determinística para micropilotes $(d=13 \mathrm{~cm})$

\begin{tabular}{|c|c|c|c|c|c|c|c|}
\hline $\begin{array}{c}\text { Long. } \\
\text { micro- } \\
\text { pilote, } \\
\text { m }\end{array}$ & $\begin{array}{c}\text { prom. } \\
\text { por } \\
\text { fuste, } \\
\text { ton } \\
\mu_{\mathrm{R}}\end{array}$ & $\begin{array}{c}\text { desv. } \\
\text { están- } \\
\text { dar, } \\
\text { ton } \\
\sigma_{\mathrm{R}}\end{array}$ & $\begin{array}{c}\text { fuste } \\
+ \\
\text { desv. } \\
\text { ton }\end{array}$ & $\begin{array}{c}\text { fuste } \\
- \\
\text { desv. } \\
\text { ton }\end{array}$ & $\begin{array}{c}\text { fuste, } \\
\text { ton } \\
\text { deter- } \\
\text { minísti- } \\
\text { ca }\end{array}$ & $\begin{array}{c}\text { punta, } \\
\text { ton } \\
\text { deter- } \\
\text { minísti- } \\
\text { ca }\end{array}$ & $\begin{array}{c}\text { Pérdida } \\
\text { de ca- } \\
\text { pacidad } \\
\text { de carga } \\
\text { por fuste, } \\
\text { \% }\end{array}$ \\
\hline 12 & 4,58 & 0,52 & 5,11 & 4,07 & 10,37 & 1,53 & 55,76 \\
\hline 20 & 12,34 & 1,91 & 14,25 & 10,43 & 28,80 & 2,55 & 57,14 \\
\hline 40 & 44,21 & 7,30 & 51,51 & 36,91 & 115,20 & 5,10 & 61,62 \\
\hline 60 & 93,23 & 13,13 & 106,37 & 80,10 & 259,21 & 7,65 & 64,03 \\
\hline
\end{tabular}

Por otro lado, los estadísticos de la Tabla 2 permiten estimar la probabilidad de falla para micropilotes de diferentes longitudes. Para tal fin se puede establecer los siguientes supuestos: a) Las funciones de densidad de probabilidad empíricas mostradas en la Figura 9 se ajustan a una variable aleatoria que se distribuye de forma gaussiana con la media de la variable aleatoria resistencia $\mathrm{R}, \mu_{\mathrm{R}} \mathrm{y}$ desviación estándar de $\mathrm{R}, \sigma_{\mathrm{R}}$. b) Se conoce del diseño estructural la variable aleatoria solicitación $\mathrm{S}$ con valor medio $\mu_{\mathrm{s}} \mathrm{y}$ desviación estándar $\sigma_{\mathrm{s}}$. c) La variable aleatoria $\mathrm{S}$ se distribuye también de forma gaussiana. d) Hay independencia estadística entre R y S. Para que no se presente la falla, se necesita que la resistencia $R$ sea mayor a la solicitación $\mathrm{S}(\mathrm{R}>\mathrm{S})$, es decir que $\mathrm{R}-\mathrm{S}>0$. La probabilidad de falla se define como la probabilidad que $\mathrm{R}$ - S sea menor que cero, $\mathrm{p}(\mathrm{R}-\mathrm{S})<0$. Con base en lo anterior se puede derivar una función de densidad de probabilidad teórica gaussiana, con media $\mu_{(\mathrm{R}-\mathrm{S})}=\mu_{\mathrm{R}}-\mu_{\mathrm{S}}$ y desviación estándar $\sigma_{(\mathrm{R}-\mathrm{S})}=\sqrt{\sigma_{\mathrm{R}}^{2}-\sigma_{\mathrm{S}}^{2}}$.

De esta manera se puede calcular la probabilidad de falla $\mathrm{p}(\mathrm{R}-\mathrm{S}<0)=\Phi\left(\mu_{(\mathrm{R}-\mathrm{S})}, \sigma_{\mathrm{S}(\mathrm{R}-\mathrm{S})}, 0\right)$, donde $\Phi$ es la función de distribución de probabilidad gaussiana (Ang y Tang, 2007). En este caso, se puede evaluar la probabilidad de falla del micropilote integrando la influencia del proceso constructivo. Probabilidades de falla para pilotes con base en funciones de densidad de tipo normal y lognormal han sido propuestas también por Haldar y Babu (2008) y Niandou y Breysse (2007).

En la Figura 10 se observa que al tener en cuenta, tanto la variabilidad inherente de los parámetros geomecánicos como el proceso constructivo de perforación para el emplazamiento del micropilote autoperforante e inyectado 
gravitacionalmente, la capacidad de carga disminuye notoriamente. Los análisis geotécnicos tradicionales usan el factor de seguridad para dividir la capacidad de carga y entregar la capacidad de carga de diseño. En este caso, la capacidad de carga determinista sobrepasa en un factor que varía desde 2 hasta 3 para longitudes de micropilote de 12 a $60 \mathrm{~m}$, respectivamente. Dicho factor se puede entender como el factor de seguridad que se le debe aplicar al análisis determinístico para tener en cuenta la incertidumbre aleatoria de las propiedades del suelo y del proceso constructivo. Lo anterior permite dar una explicación racional al factor de seguridad, situación que mejora ostensiblemente el carácter subjetivo de factores sugeridos en códigos de diseño.

Adicionalmente, la Figura 10 hace las veces de carta de diseño de un micropilote autoperforante e inyectado de 13 $\mathrm{cm}$ de diámetro para un estrato homogéneo con ángulo de fricción $\phi^{\prime}=20^{\circ}$. La utilidad práctica se observa en que el análisis estructural de una edificación entrega la carga que se debe transmitir al suelo a través del micropilote. Con este dato se ingresa por las abscisas de la Figura 10 y el resultado es la longitud del micropilote tipo Anker que satisface dichos requerimientos.

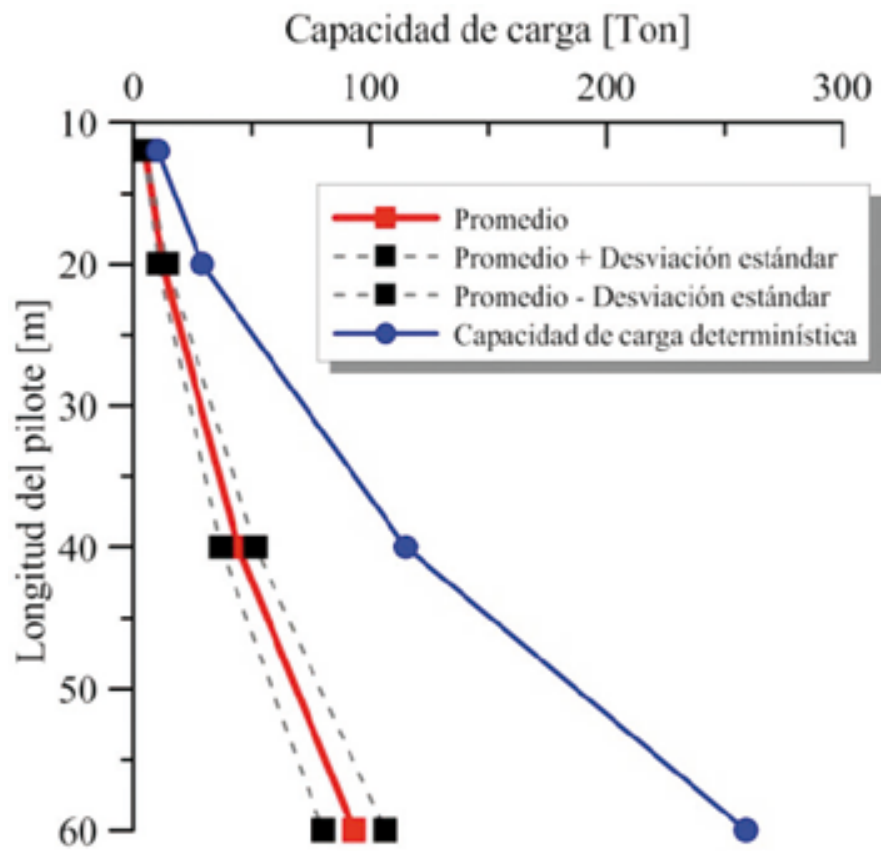

Figura 10: Capacidad de carga por fuste para diferentes longitudes de micropilote. Análisis estocástico teniendo en cuenta el proceso constructivo. Análisis determinísticos con valores medios.

\section{Conclusiones}

El presente artículo muestra un procedimiento para incorporar la incertidumbre en los parámetros geomecánicos y el proceso constructivo en el cálculo de la capacidad de carga de un micropilote autoperforante e inyectado gravitacionalmente. El análisis de elementos finitos aleatorio permite que a partir de unas funciones de distribución de parámetros del suelo, se obtenga probabilidades de falla del sistema. La simulación de Montecarlo de la excavación con elementos finitos entrega insumos para integrar la probabilidad de desplazamiento de la pared de la excavación en un análisis basado en confiabilidad de la capacidad de carga de micropilotes fundamentado en el teorema de la probabilidad total. Adicionalmente se propone un procedimiento para estimar la probabilidad de falla de un micropilote integrado con la influencia del proceso constructivo.

Se encuentra que la aplicación de un factor de seguridad entre dos y tres al análisis de carga por fuste para micropilotes autoperforantes e inyectados, es capaz de cubrir la influencia de la variabilidad de los parámetros geomecánicos y la disminución de la capacidad de carga por el proceso constructivo. Por lo anterior, se da una explicación racional al factor de seguridad que se debe aplicar para obtener la capacidad de carga de diseño. Esta situación mejora ostensiblemente el carácter subjetivo de factores sugerido en códigos de diseño. Futuras investigaciones deben considerar comparar estos resultados con los obtenidos en pruebas de carga in situ de micropilotes Anker para demostrar si la capacidad de carga medida es consistente con la estimada con este análisis. Además se puede contemplar la exhumación de micropilotes para medir los diámetros obtenidos.

\section{Agradecimientos}

El primer y tercer autor agradecen a la empresa SOLOTRAT, al grupo de investigación en fundaciones, ensayos de campo y estructuras de contención de la Universidad de Brasilia (GPFees) por el soporte técnico en el desarrollo de la presente investigación y al Consejo Nacional de Desarrollo Científico y Tecnológico (CNPq) por el apoyo financiero. El segundo autor agradece a la Pontificia Universidad Javeriana por el soporte económico 
a la presente investigación con el proyecto 00004711 Estudio experimental de la licuación por flujo. Los autores agradecen al MOX de la Universidad de Los Andes Bogotá - por el soporte computacional para la realización del presente proyecto.

\section{Referencias}

Ang, A. and Tang, W. (2007). Probability concepts in engineering. $2^{\text {nd }}$ Edition. John Wiley and Sons

Baecher, G. and Christian, J. (2003). Reliability and statistics in geotechnical engineering. John Wiley and Sons

Baroth, J. and Malecot, Y. (2010). Probabilistic analysis of the inverse analysis of an excavation problem. Computers and Geotechnics 37, 391-398

Burland, J.B. (1973). Shaft friction of piles in clay - a simple fundamental approach. Ground Engineering 6(3), 30-42

Cherubini, C. (2000). Probabilistic approach to the design of anchored sheet pile walls. Computers and Geotechnics 26, 309-330

Delgado, M. (2001). Ingeniería de fundaciones. Editorial Escuela Colombiana de Ingeniería

Fellin, W., King, J., Kirsch, A. and Oberguggenberger, M. (2010). Uncertainty modelling and sensitivity analysis of tunnel face stability. Structural Safety 32, 402-410

FHWA (2000). Micropile design and construction guidelines. Implementation Manual. USA Department of Transportation, Federal Highway Administration

Griffiths, D., Fenton, G. and Tveten, D. (2005). Probabilistic passive earth pressure analysis by the random Finite Element Method. Prediction, analysis and design in geomechanical applications. Proceedings of the Eleventh International Conference on Computer Methods and Advances in Geomechanics, Torino, Italy, 19-24 June, Vol. 4.

Haldar, S. and Babu, S. (2008). Effect of soil spatial variability on the response of laterally loaded pile in undrained clay. Computer and Geotechnics 35, 537-547

Harahap, I. and Wong, C. (2008). Soil parameters and bearing capacity derived from responses of drilled shaft socketed into rock. Proceedings of International Conference of Construction and Building Technology E (32), 391-402

Helwany, S. (2007). Applied Soil Mechanics with ABAQUS Applications. 1st edition. John Wiley and Sons

Huang, J., Griffiths, D. and Fenton, G. (2010). Probabilistic analysis of coupled soil consolidation. Journal of Geotechnical and Geoenvironmental Engineering 136 (3), 417-430

Kamruzzaman, M. and Lye, L. (2008). Probabilistic Analysis of Laterally Loaded Pile-Soil System using Monte Carlo Simulation. Proceedings of the Eighth ISOPE Pacific/Asia Offshore Mechanics Symposium. Bangkok, Thailand, November 10-14

Kuo, Y., Jaksa, M., Kaggwa, W., Fenton, G., Griffiths, D. and Goldsworthy, J. (2004). Probabilistic analysis of multi-layered soil effects on shallow foundation settlement. Proceedings in $9^{\text {th }}$ Australia-New Zealand Conference on Geomechanics, Auckland, New Zealand

Luo, Z., Atamturktur, S., Yuanqiang, C. and Hsein Juang, C. (2012). Reliability analysis of basal-heave in a braced excavation in a 2-D random field. Computers and Geotechnics 39, 27-37

Mendoza, C.C., Ruge, J., Cunha, R. P. y Lizcano, A. (2011). Comportamiento mecánico de excavaciones de pequeño diámetro para suelo estructurado "pilote Alluvial Anker". $14 a$ Conferencia Panamericana de Mecánica de Suelos e Ingeniería Geotécnica. Toronto, Ontario, Canadá

Niandou, H. and Breysse, D. (2007). Reliability analysis of a piled raft accounting for soil horizontal variability. Computers and Geotechnics 34, 71-80

Papaioannou, I. and Straub, D. (2012). Reliability updating in geotechnical engineering including spatial variability of soil. Computers and Geotechnics 42, 44-51

Phoon, K.K. and Kulhawy, F. (1999). Characterization of geotechnical variability. Canadian Geotechnical Journal 4 (36), $612-624$

Poulos, H.G. and Davis, E.H. (1980). Pile foundation analysis and design. 1st edition. John Wiley and Sons

Prada, L.F., Ramos, A., Solaque, D. y Caicedo, B. (2011). Confiabilidad aplicada al diseño geotécnico de un mutro de contención. Obras y Proyectos 9, 49-58

Sentz, K., and Ferson, S. (2011). Probabilistic bounding analysis in the quantification of margins and uncertainties. Reliability Engineering and System Safety 96 1126-1136

Tandjiria, V., Teh, C. and Low, B. (2000). Reliability analysis of laterally loaded piles using response surface methods. Structural Safety 22, 335-355

Wang, G. and Sitar, N. (2011). Static and dynamic axial response of drilled piers. II Numerical simulation. Journal of Geotechnical and Geoenvironmental Engineering 12 (137), 1143-1153

Zevgolis, I. and Bourdeau, P. (2010). Probabilistic analysis of retaining walls. Computers and Geotechnics 37, 359-373 